\title{
A REPORT OF 12 SELECTED CASES OF PULMONARY HISTOPLASMOSIS
}

\author{
BY \\ ALEX SALIBA AND OREN A. BEATTY \\ From District Two State Tuberculosis Hospital, Louisville, Kentucky, U.S.A.
}

(RECEIVED FOR PUBLICATION APRIL 6, 1960)

Once considered a rare and fatal disease, histoplasmosis is now increasingly suspected or diagnosed. There may be two apparent reasons for this; first, it is possible that pulmonary histoplasmosis has always been prevalent, but increase in knowledge and modern diagnostic techniques have rendered it more readily recognizable. A similar argument was once put forth in relation to the apparent increase in carcinoma of the lung. Secondly, and this is more likely, the indiscriminate use of wide-spectrum antibiotics and the increasing use of steroids could possibly have altered the balance of normal host inhabitants and prepared the ground for infection by the mycoses. Schwarz and Baum (1957) presented a historical background pointing out the more recent important contributions in this field by Palmer, Christie, Furcolow, Emmons, Puckett, Straub, Ajello, and others.

The following cases have been selected for presentation because of the varying radiological appearances, which under ordinary circumstances are not distinguishable from pulmonary tuberculosis. Four patients were treated with amphotericin B and resectional surgery. Two received surgery but no medical therapy; four received medical therapy without surgery. One patient was not actively treated because positive mycology was not obtained in the presence of a highly positive complementfixation reaction. The last patient died within three days of admission to this hospital before any therapy could be instituted. Of the surgical cases, three had post-operative air pockets, one of which required thoracoplasty. Two of the patients are white women and 10 white men. Eight were in the group 40-57 years and four in the 23-39 years old. Farming was the occupation of five $3 f$ them.

\section{MATERIAI}

CASE 1.-C.B., a 37-year-old white man, was first admitted to this institution in July, 1954. His occupation was that of a farm labourer. His symptoms, namely, loss of weight, malaise, haemoptysis, dated back to January of the same year. A chest radiograph showed a cavity in the left upper lobe, and one sputum was reported positive for acid-fast bacilli on smear, but this was not confirmed by culture. This patient was in hospital for 323 days and treated as tuberculous by bed rest, daily isoniazid, and intermittent streptomycin and pneumoperitoneum. Throughout his stay in hospital sputum examinations remained negative for tuberculosis by repeated smears and culture. He suffered a relapse in the latter part of 1958 and was readmitted in November (Fig. 1). The diagnosis of pulmonary histoplasmosis was then established with a positive histoplasmin skin test, positive complement fixation, and several sputum examinations from which were cultured Histoplasma capsulatum. The patient received three weeks of intravenous amphotericin B therapy and a segmental resectio $\Phi$ of the left upper lobe was carried out. He then received $Q$ further course of amphotericin B therapy and the posto operative course was uneventful. His more recent out patient follow-up radiographs are essentially clear apart from evidence of surgery on the left side.

This is a case of a solitary cavity that was assumed to be of tuberculous origin in spite of repeated negative bacteriological findings. The diagnosis of pulmonary histoplasmosis was eventually proved, and it is noteworthy that the cavity did not close after amphotericin B therapy, and also in spite of the pneumoperitoneum the patient had previously received. Subsequent cultures for histoplasmosis have all been negative. The follow-up period is six months.

CASE 2.-D. B., a 23-year-old white woman, had been working in our hospital laboratory for approximately one year. Her general condition had always been very good and she had an uneventful confinement in July, 1959. After about 10 weeks she returned to work and noticed a protracted cold with cough followed by a rightsided pleuritic type of pain. Physical examination was not remarkable. There was no previous record of a skin test as far as the patient could recall. A pre-employment radiograph at this hospital in September, 1958, was essentially normal. Six months later, in March, 1959, a repeat radiograph showed a smooth round density just off the right hilum, but the patient was entirely symptom free. She was observed for a further six months when another radiograph was obtained in September, 1959 


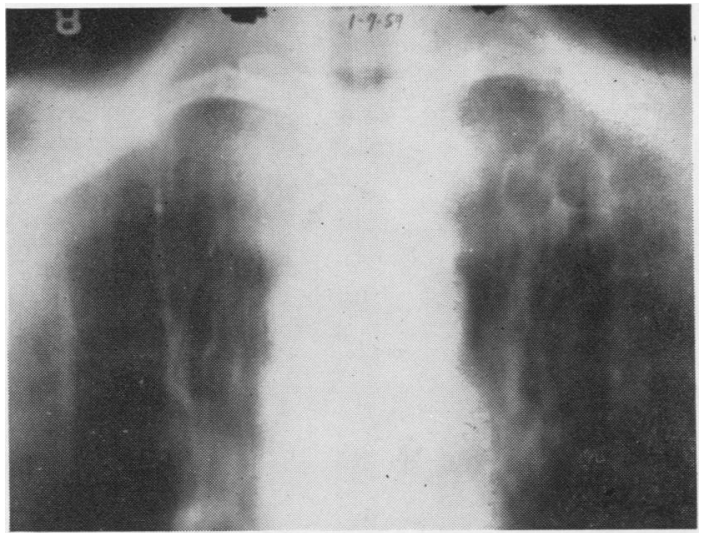

FIG. 1.-Tomogram (Case 1) of January, 1959, confirming the presence of multilocular cavitation in left upper lobe.

(Fig. 2). This showed that the lesion was slightly larger and coincided with the onset of symptoms. Skin sensitivity showed positive reaction to P.P.D. intermediate strength and histoplasmin, and complement fixation was also highly positive for histoplasmosis. She later had a thoracotomy in another hospital, where several glands were removed, and these were reported to be " typical of tuberculosis." Several cultures from caseous material were negative for tuberculosis. However, a section of the specimen was submitted to another centre, and Histoplasma capsulatum was found in the tissue. This patient did not receive any amphotericin B therapy and is being kept under careful observation.

This is a case of progressive primary pulmonary histoplasmosis that was relatively asymptomatic until after the patient's confinement. No gross parenchymal disease was found at the time of surgery. However, she was not covered with amphotericin B therapy, and it remains to be seen if a "cure" can be achieved in such an early case, or if she is likely to suffer a relapse.

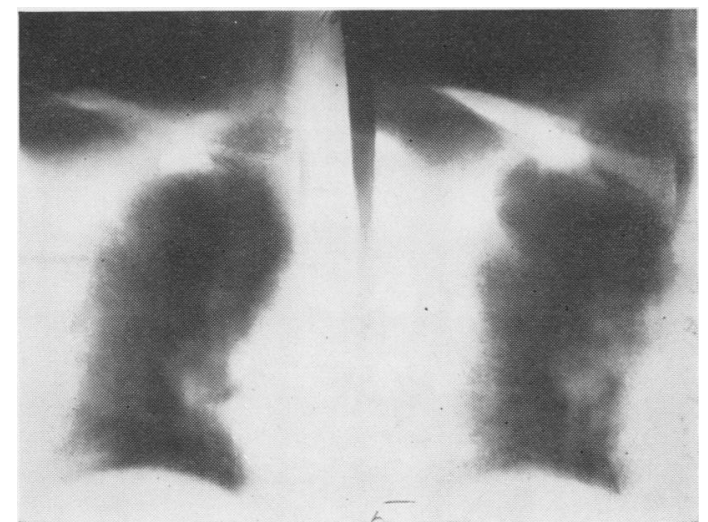

FIG. 2.-Tomogram (Case 2) showing the enlarged glands at the right hilum.
CASE 3.-W. S. was a 57-year-old white man, a railroad worker. Pulmonary disease was first picked up approximately two years ago when the patient was complaining of weakness, drowsiness and a general feeling of ill health. He was treated by his physician at home for several months. The patient continued to feel out of sorts and was losing weight, and a further radiograph showed some deterioration in pulmonary pathology. He was admitted here in September, 1959. Sputum examinations were negative for tuberculosis, and complementfixation reaction was positive for histoplasmosis. A chest film showed nodular type disease with some calcification, mostly in both upper lobes. Tomography showed cavitation in the left apex. This patient was started on a therapeutic trial of antituberculous drugs consisting of daily streptomycin and isoniazid which appeared to make no impression on the disease. At the end of November reports of sputum results positive for histoplasmosis were received, and amphotericin $\mathbf{B}$ therapy was begun intravenously. This course has not been completed, and the patient is still in the hospital.

This is a case of bilateral nodular disease with cavitation, from which Histoplasma capsulatum was eventually isolated and appropriate therapy was instituted.

CASE 4.-G. G., a 57-year-old white man, a labourer, in 1957 developed a protracted cold which did not respond to treatment by his family physician. On $x$-ray examination the patient was suspected of having pulmonary tuberculosis, but in view of negative bacteriology was just kept under observation in the out-patient department of a sanatorium. The original chest film in July, 1957, showed scattered disease throughout the right lung with pleural thickening. Minimal involvement of the left apex was also suspected. By December, 1957, there had been some increase in the area of disease in the left upper lobe. Radiographs on admission here in July, 1959, showed extensive consolidation and cavitation throughout both lung fields (Fig. 3). Multiple cavities were present on both sides. On admission here the patient was in a terminal state and died within three days before any treatment was instituted. At necropsy both lungs were extensively shrunken and destroyed with multiple cavitation. Histoplasma capsulatum was found in tissue sections, but no acid-fast bacilli by smear or culture were recovered.

This represents an advanced case of histoplasmosis that was followed up for two years without any treatment and proved rapidly progressive and fatal.

CASE 5.-A. B., a 32-year-old white man, a truck driver, was first diagnosed as tuberculous in February, 1957. He was treated for several months in another sanatorium and subsequently transferred here in July, 1957. His chest film showed a partial contraction of the left upper lobe with cavitation. Numerous sputum examinations were negative for tuberculosis by smear and culture, and the complement-fixation reaction for histoplasmosis was positive. A left upper lobectomy was carried out in August, 1957. Examination of this 
specimen showed the presence of Histoplasma capsulatum. Post-operatively an air pocket developed and persisted in spite of repeated fluid and air aspirations. A month's course of amphotericin B therapy was given intravenously, and the patient was discharged. $\mathrm{He}$ was readmitted in September, 1959, because a nodular lesion in the right upper lobe on follow-up examination was found to have become excavated. The left apical pocket persisted and contained a fluid level. On readmission here the complement-fixation reaction for histoplasmosis was still positive, but repeated sputum examinations were negative both for tuberculosis and for histoplasmosis. A further four months' course of amphotericin B therapy intravenously was begun and has now been completed. The left apical air pocket closed; however, the cavity in the right upper lobe persisted. This patient has declined to accept further surgery for the right-sided cavity, and has been discharged.

This case was diagnosed only post-operatively and, therefore, did not receive any amphotericin B therapy pre-operatively. As with other cases, it is noteworthy that an air pocket developed and persisted; however, it responded satisfactorily to amphotericin B therapy. Again this case shows the lack of response of the cavity on the right side to amphotericin B.

CASE 6.-E. R., this 48-year-old white man, a farmer, was first seen in the out-patient department of this hospital in Cctober, 1957. Chest radiographs showed a giant cavity in the left upper lobe area with surrounding infiltration. Some aspiration spread to the right upper lung field had also taken place. Sputum examinations were positive for Mycobacterium tuberculosis on admission. However, the histoplasmin skin test was also positive, and the complement-fixation test was strongly positive for histoplasmosis. Accordingly sputum examinations for fungi were submitted, and several of these were reported positive for Histoplasma capsulatum. Therefore, on admission the patient was started on antituberculous therapy consisting of daily isoniazid and P.A.S., and subsequently the isoniazid was increased to a full high dosage $(16 \mathrm{mg} . / \mathrm{kg}$.). In December, 1958, amphotericin B therapy intravenously was begun and was given for four months. This patient's sputum converted both for tuberculosis and for histoplasmosis. A pneumoperitoneum was also given. Subsequent $x$-ray examinations showed a considerable improvement on the right side and shrinking of the left apical cavity with clearing of the surrounding infiltration. However, tomography in July, 1959, confirmed the persistence of the cavity at the left apex, though much reduced in size. The complement-fixation reaction remained highly positive for histoplasmosis. Resectional surgery on the left side was advised, but the patient declined to accept it. He has been discharged and has been followed up in the out-patient department for the past eight months.

This is a case of cavitary disease that has been proved to be active histoplasmosis combined with active tuberculosis. Again some response is noted following therapy, but the cavity failed to close.
CASE 7.-L. C., a 51-year-old white woman, a housewife, had symptoms dating back to December, 1957, when she developed influenza-like symptoms which improved. Subsequently, however, she became short of breath and had a productive cough with right-sided chest pain and a high temperature. She was referred to this hospital in January, 1958, for diagnosis and treatment. She was suspected as a possible case of faradvanced, pulmonary tuberculosis, but sputum examinations were negative for tuberculosis. Chronic suppurative pneumonia was a second possibility in the differential diagnosis. She was treated with wide-spectrum antibiotics and daily isoniazid and P.A.S., but left the hospital against medical advice after 51 days. She continued on the antituberculous drugs while at home. She had been offered surgery which she refused while in hospital. On re-evaluation at this hospital on readmission in March, 1959 , she was found to have had numerous sputum examinations negative by smear and culture for tuberculosis, but a skin test was positive for histoplasmin as well as for P.P.D. A complement-fixation reaction was highly positive for histoplasmosis. Sputum examinations were submitted, and several of these were found positive for Histoplasma capsulatum. A chest film showed multiple cavities in the right upper lobe with a spread to the right lower lobe (Fig. 4). She was given a preoperative course of amphotericin B, following which resectional surgery was carried out on the right side. A further course of amphotericin B was given posto operatively. The total dosage received was $1,298 \mathrm{mg}$ Post-operatively a bronchopleural fistula developed wit a persistent air pocket which necessitated a thoracoplast Histoplasma capsulatum was recognized on sections of the surgical specimen. Subsequent sputum examina- $\mathbb{\perp}$ tions have been negative for Histoplasma capsulatum, and $\overrightarrow{\vec{F}}$ the complement-fixation reaction is still positive though not to the high degree it had been pre-operatively.

This is a case of cavitary pulmonary histoplasmosis in which the diagnosis was established pre-operatively.

CASE 8.-P. P., a 54-year-old white man, a railroad worker, had a history going back to June, 1953, with 3 . symptoms of bronchitis and asthma, necessitating repeated admissions to hospital and treatment by numer- $\frac{\rho}{3}$ ous physicians. All sputum examinations were negative for acid-fast bacilli. A tuberculin skin test was also 옥 negative. The patient was found to have a complement- $D$ fixation reaction positive for histoplasmosis and was referred to this hospital in September, 1958, for further evaluation and treatment. A chest film in 1954 showed a $\sigma$ small lesion in the left upper lobe area, and both lung N fields were emphysematous but relatively clear. In 1957, N a radiograph showed a slight increase in the lesion in the $\omega$ left upper lobe and new lesions in the right apex. $A$ chest film in July, 1958, revealed extensive bilateralco increase in the disease process with cavitation on the right. A chest film on admission here was essentially $\stackrel{?}{+}$ unchanged, and a tentative diagnosis of pulmonary tuberculosis was made. However, sputum examinations ${ }_{0}^{\circ}$ were repeatedly negative by smear and culture. Sputum $\mathbb{D}$ 


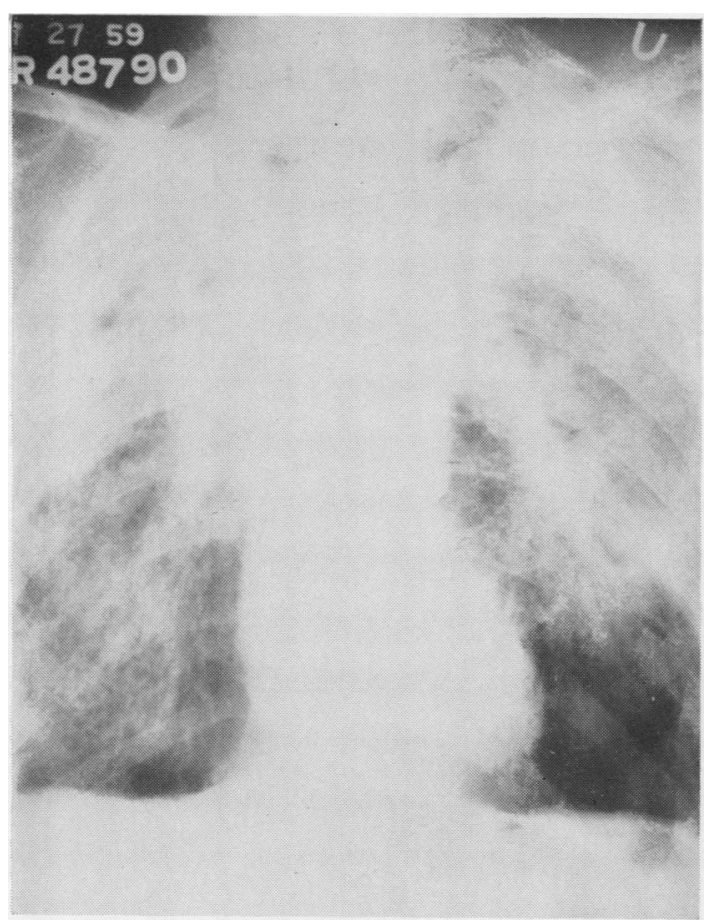

Fig. 3.-Chest film (Case 4) showing extensive involvement of both lungs with bilateral cavitation.

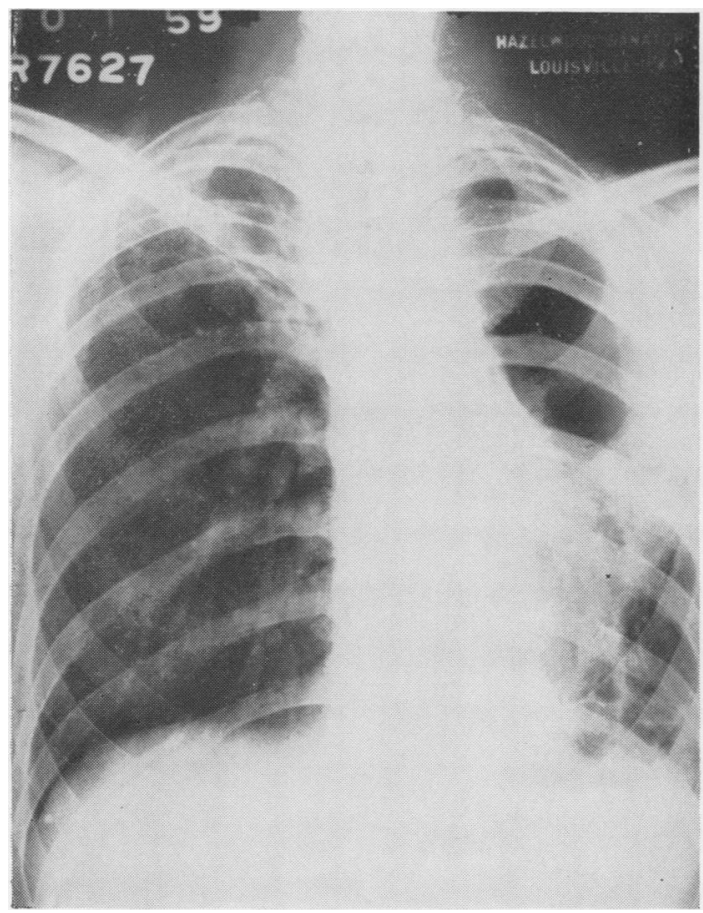

FIG. 5.-Chest film (Case 9) showing a destroyed left lung with huge cavitation. Spread has taken place to the right upper lobe.

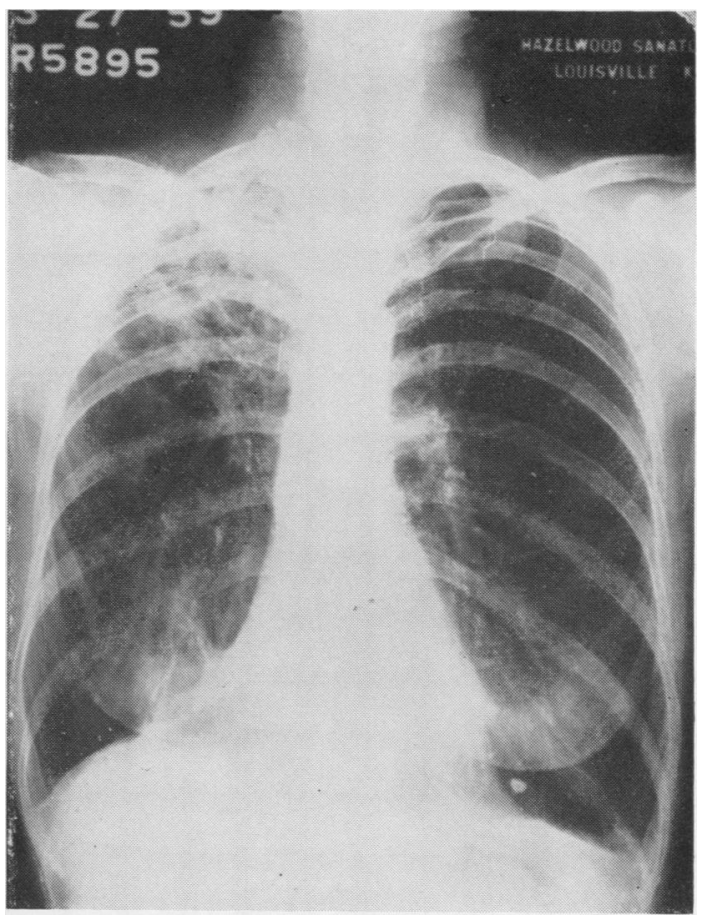

Fig. 4.-Chest film (Case 7) showing a contracted right upper lobe with cavitation and spread to right lower lobe.

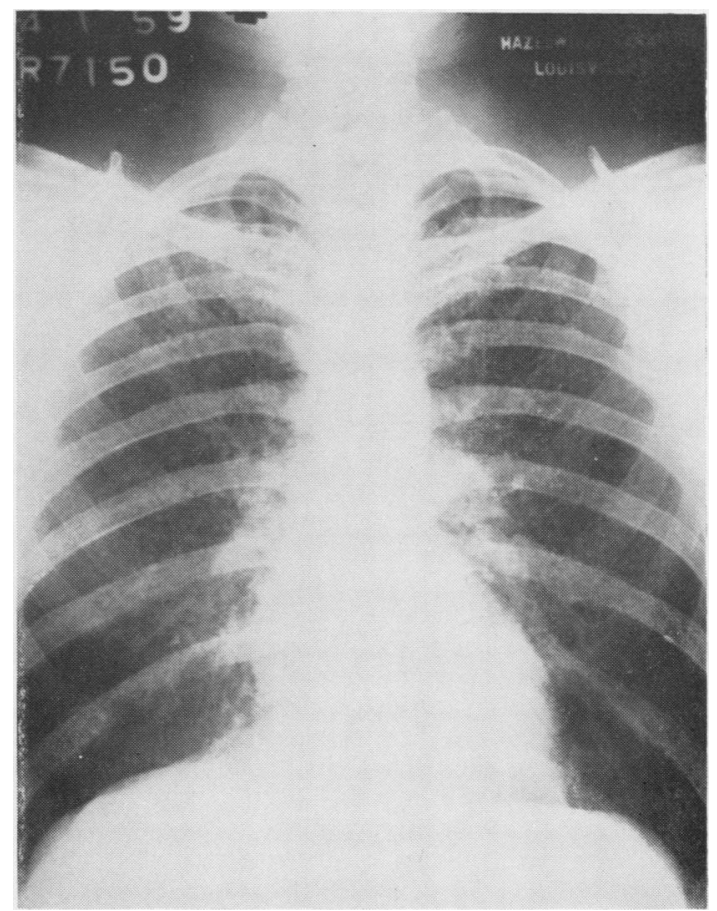

FIG. 6.-Chest film (Case 12) showing miliary type nodules bilaterally, more conglomerate towards apices. 
TABLE

SUMMARY OF CASES REVIEWED

\begin{tabular}{|c|c|c|c|c|c|c|c|c|c|c|c|c|}
\hline \multirow{2}{*}{$\begin{array}{l}\text { Case } \\
\text { No. }\end{array}$} & \multirow{2}{*}{$\begin{array}{l}\text { Race and } \\
\text { Sex }\end{array}$} & \multirow{2}{*}{ Age } & \multirow{2}{*}{$\begin{array}{l}\text { Skin } \\
\text { T.B. }\end{array}$} & \multirow{2}{*}{$\begin{array}{c}\text { Sensi- } \\
\text { tivity } \\
\text { Histo- } \\
\text { plas- } \\
\text { mosis }\end{array}$} & \multicolumn{2}{|c|}{$\begin{array}{l}\text { Complement } \\
\text { Fixation for } \\
\text { Histoplasmosis }\end{array}$} & \multicolumn{2}{|c|}{$\begin{array}{c}\text { Sputum Culture } \\
\text { Initially }\end{array}$} & \multirow{2}{*}{ Surgery } & \multirow{2}{*}{$\begin{array}{l}\text { Course and } \\
\text { Complications }\end{array}$} & \multirow{2}{*}{\multicolumn{2}{|c|}{$\begin{array}{l}\text { Tissue } \\
\text { Section }\end{array}$}} \\
\hline & & & & & I & II & T.B. & $\begin{array}{l}\text { Histo- } \\
\text { plas- } \\
\text { mosis }\end{array}$ & & & & \\
\hline $\begin{array}{r}1 \\
2 \\
3 \\
4 \\
5 \\
6 \\
7 \\
8 \\
9 \\
10 \\
11 \\
12\end{array}$ & $\begin{array}{ll}\mathbf{W} & \mathbf{M} \\
\mathbf{W} & \mathbf{F} \\
\mathbf{W} & \mathbf{M} \\
\mathbf{W} & \mathbf{M} \\
\mathbf{W} & \mathbf{M} \\
\mathbf{W} & \mathbf{M} \\
\mathbf{W} & \mathbf{F} \\
\mathbf{W} & \mathbf{M} \\
\mathbf{W} & \mathbf{M} \\
\mathbf{W} & \mathbf{M} \\
\mathbf{W} & \mathbf{M} \\
\mathbf{W} & \mathbf{M}\end{array}$ & $\begin{array}{l}37 \\
23 \\
57 \\
57 \\
32 \\
48 \\
51 \\
54 \\
39 \\
51 \\
42 \\
40\end{array}$ & $\begin{array}{l}\overline{+} \\
+ \\
+ \\
+ \\
+ \\
+ \\
+ \\
+ \\
+\end{array}$ & $\begin{array}{l}+ \\
+ \\
- \\
+ \\
+ \\
+ \\
+ \\
+ \\
+ \\
+ \\
+\end{array}$ & \multicolumn{2}{|c|}{\begin{tabular}{l|l}
$1 / 32$ & $1 / 16$ \\
$1 / 16$ & $1 / 128$ \\
- & $1 / 32$ \\
$1 / 32$ & $1 / 16$ \\
$1 / 16$ & $1 / 32$ \\
$1 / 128$ & $1 / 32$ \\
$1 / 64$ & $1 / 128$ \\
$1 / 16$ & $1 / 64$ \\
$1 / 64$ & $1 / 256$ \\
\multicolumn{2}{c}{ None } \\
\multicolumn{1}{c}{ Non }
\end{tabular}} & $\begin{array}{l}-\mathbf{N} \\
-\mathbf{N} \\
- \\
+ \\
- \\
- \\
- \\
-\end{array}$ & $\begin{array}{c}+ \\
+ \\
+ \\
+ \\
+ \\
+ \\
+ \\
+ \\
+ \\
+\end{array}$ & $\begin{array}{l}\text { Yes } \\
\text { No } \\
\text { Ÿes } \\
\text { No } \\
\text { Yes } \\
\text { No } \\
\text { Yes } \\
\text { No treat- } \\
\text { ment }\end{array}$ & $\begin{array}{l}\text { Discharged } \\
\text { In hospital } \\
\text { Died. Necropsy } \\
\text { B-p fistula. Discharged } \\
\text { Discharged } \\
\text { B-p fistula. Discharged } \\
\text { Discharged } \\
\text {," } \\
\text { B-p fistula. Discharged } \\
\text { Discharged }\end{array}$ & $\begin{array}{l}\text { Negat } \\
H . \text { cal }\end{array}$ & $\begin{array}{l}\text { atum } \\
\text {,, } \\
\text {,, }\end{array}$ \\
\hline
\end{tabular}

examinations for fungi were submitted, and several of these were found positive for Histoplasma capsulatum. Amphotericin B therapy was started in November, 1958. This treatment was poorly tolerated, and only 18 intravenous administrations had been given by March, 1959, when therapy was discontinued. Subsequent follow-up radiographs showed marked improvement in the bilateral disease. Tomography in April, 1959, showed definite residual thin-walled cavities in both upper lobes. Subsequent sputum examinations have been negative for fungi. The follow-up on this patient is nine months.

This is another case of chronic bilateral pulmonary histoplasmosis with cavitation and emphysema from which the patient has become a respiratory cripple. Some radiological improvement was obtained after treatment with amphotericin $\mathbf{B}$, but cavitation persisted.

CASE 9.-T. H., a 39-year-old white man, a farmer, was first picked up in 1956 by a routine chest radiograph. He was found to have bilateral cavitary disease and was put on antituberculous chemotherapy. However, sputum examinations were negative for tuberculosis. $\mathrm{He}$ was referred to this hospital in September, 1959, for further evaluation. Skin tests were negative both for P.P.D. and histoplasmin. Repeated sputum examinations were negative by smear and culture for tuberculosis. Complement fixation was positive for histoplasmosis and sputum examinations were submitted for special fungus studies and Histoplasma capsulatum was isolated. A chest film on admission showed a giant cavity occupying the upper two-thirds of a contracted left lung (Fig. 5). The lower portion of the lung was grossly infiltrated and fibrotic. On the right side a small amount of infiltration was seen in the upper third of the lung field. On admission here the patient was started on streptomycin daily with high dosage of isoniazid pending further studies. Following reports of cultures positive for Histoplasma capsulatum, amphotericin B therapy was started in October, 1959, and a course of four months was completed. Symptomatically the patient's productive cough and his sense of wellbeing have improved considerably. More recent sputum examinations have been negative for fungi, though the radiological appearances have not changed considerably. This patient is a poor surgical risk, and he has been discharged.

This is a chronic, far-advanced case of pulmonary histoplasmosis with an excavated, destroyed lefo lung. Again, in spite of symptomatic improvements and conversion of sputum, not much impressiofi has been made on this type of disease by amphotericin B.

CASE 10.-C. L., a 51-year-cld white man, a farmer, had symptoms dating back to early 1958 and consisting of malaise, dyspnoea, and loss of appetite. He was admitted to a sanatorium in September, 1958, with a presumptive diagnosis of far-advanced, pulmonary tuberculosis and placed on therapy consisting of daily isoniazid and P.A.S. Sputum examinations were negative for tuberculosis and for fungi. In November, 1958, he was transferred to our hospital for possible surgery. Because of negative P.P.D. and a positive histoplasmin skin test, and also because sputum examinations were repeatedly negative for tuberculosis by smear and culture, surgery was delayed, and the patient was maintained on isoniazid and P.A.S. daily. Further sputum examinations for fungi were also negative. Chest film on admission showed a moderate amount of disease with cavitation and a fluid level in the left upper of lobe. On the right side a minimal amount of infiltration was present in the right upper lobe area and the rest of the lung fields were clear. Subsequent follow-up radiographs showed essentially no change, and on March, 1959, a segmental resection of the left upper lobe was carried out. Sections of this specimen were submitted for special studies and Histoplasma capsulatum was found. Amphotericin B was not given to this patient.

This is an example of a presumptive diagnosis of ${ }^{\circ}$ advanced tuberculosis, but no bacteriological proof 
was obtained. Antituberculous therapy failed to make any impression on the disease. Though histoplasmosis was suspected, mycological proof by sputum examinations could not be obtained. The diagnosis was established post-operatively.

CASE 11.-O. B., a 42-year-old white man, a road worker, was diagnosed as a case of far-advanced pulmonary tuberculosis in 1956, and was admitted to a sanatorium. His symptoms at the time consisted of a productive cough, one episode of haemoptysis, and subsequently some blood streaking, easy fatigue, and slight loss of weight. Sputum examinations were negative for acid-fast bacilli, and the patient was placed on antituberculous chemotherapy. He was transferred to this hospital in September, 1958, for surgical evaluation. A chest film on admission showed the area of disease mainly involving both upper lobes, more so on the left side. Cavitation was present at both apices. Comparison with previous films going as far back as 1956 showed some improvement in the bilateral disease, but cavitation persisted. Skin tests were positive for P.P.D. and histoplasmin. The patient was continued on antituberculous chemotherapy and in the meantime sputum examinations were submitted for fungus studies, as no acid-fast bacilli were found. Several sputum examinations were found positive for Histoplasma capsulatum. In November, 1958, a left upper lobectomy was carried out under amphotericin cover. Post-operatively the patient developed an air pocket which responded to amphotericin B therapy. This treatment was given for a total of $2,265 \mathrm{mg}$. in two separate courses after which he left the hospital as he did not wish to continue treatment. He has failed to return for out-patient check-ups and his present status is not known.

This is another case of chronic pulmonary histoplasmosis previously treated as tuberculosis. The diagnosis was established by sputum cultures and amphotericin B treatment was given before and after surgery. Again it is noted that a post-operative air pocket persisted which responded satisfactorily to r.edical therapy.

CASE 12.-H. J., a 40-year-old white man, a farmer, was apparently asymptomatic and was admitted here in April, 1959, when pulmonary disease was discovered following a routine mobile chest radiograph. His chest film on admission showed small nodular lesions disseminated throughout both lung fields with some calcification (Fig. 6). Subsequent tomograms showed conglomerate lesions towards both apices. Follow-up radiographs in hospital showed essentially no change following a trial of antituberculous drugs. Skin tests were positive for P.P.D. and histoplasmin. Numerous sputum examinations were negative by smear and culture for tuberculosis and for fungi. However, on admission the complement-fixation test was strongly positive for histoplasmosis, and this remained highly positive on two subsequent examinations. A wedge resection for biopsy purposes was recommended, but the patient declined and left the hospital.
This is an example of chronic pulmonary histoplasmosis, haemotogenous type, not proved by mycology but showing a highly positive complementfixation test. As diagnosis and activity could not be definitely established, amphotericin B therapy was withheld.

\section{Discussion}

Histoplasmosis was first reported by Darling in 1906 , but subsequently remained practically unrecognized. Indeed, it has been stated that until 1935 diagnosis was usually established at necropsy. Since then it has increased in importance, and Bunnell and Furcolow (1948) and Johnson and Batson (1948) described the chronic pulmonary form of histoplasmosis. Following this the number of cases reported in the literature has been increasing and more frequently these reports are from the U.S.A., although cases have been described from most countries in the world.

Awareness by physicians of the possibility of this disease is essential for diagnosis. It can mimic many other conditions and hence can be readily missed. The chronic form is indistinguishable from pulmonary tuberculosis both symptomatically and radiologically, so that accurate diagnosis rests entirely on laboratory data. Furcolow (1958) pointed out that the prevalence of tuberculous infection led to the erroneous conclusion that almost all chronic pulmonary conditions were tuberculous. This was further aided by the prevalence of positive tuberculin skin tests. It has now been estimated that there is a high incidence of reactors to histoplasmin, in some parts of the U.S.A. as high as $80 \%$ of the population. Thus a positive histoplasmin reaction in a patient with a negative tuberculin reaction can be of definite significance. For years a diagnosis of tuberculosis has been made on radiographic appearances alone, and patients have been admitted to hospital and given prolonged treatment when positive bacteriology was not obtained. In recent years we have seen an important change in tuberculosis diagnosis in that the emphasis has been switched from radiography to bacteriology. The increasing recognition of histoplasmosis and other conditions has certainly justified this move.

Because the chronic form of this disease has hitherto been readily treated as "tuberculosis," these cases are more frequently seen in sanatoria. In this institution the cases were eventually diagnosed and treated as part of a U.S. Public Health Service investigation into the therapy of the mycoses, in which we participate. In a number of instances one is extremely suspicious of histoplasmosis because of 
a positive complement-fixation reaction, and yet the particular fungus cannot be isolated from secretions. It is thus becoming more obvious that greater importance may yet be attached to a highly positive complement-fixation reaction. For instance, a positive result of 1 in 32 or higher dilution may be enough to justify a course of amphotericin B.

Various types of pulmonary histoplasmosis have been described by Rubin, Furcolow, Yates, and Brasher (1959) such as acute, disseminated, and chronic pulmonary. The chronic pulmonary form is the one usually seen in our hospital and other similar institutions. The exception in this report is that of Case 2 which represents a primary infection at the stage of progression as evidenced by the fine nodular disseminations to the pleura and pericardium seen at operation. The chronic form is less responsive to treatment, and Saliba, Beatty, and Pakalns (1960) have shown that collapse measures, such as pneumoperitoneum, apparently have no effect on mycotic cavities unlike the good results usually obtained with tuberculous cavities. Also amphotericin B appears to have no beneficial effect on pulmonary cavitary disease due to histoplasmosis.

Conrad, Saslaw, and Atwell (1959) state that probably amphotericin B should be reserved for selected cases only because of the side-effects. On the other hand, many feel that definite clinical improvement is noted with treatment, and, like others (Yates, Atay, Langeluttig, Brasher, and Furcolow, 1960), we have found that all patients so treated have had sputum conversion. These authors also state that cortisone is contraindicated. This point has not been proven as yet (provided chemotherapy cover is also given). We have used hydrocortisone with our intravenous amphotericin B therapy and have found this successfully eliminates many side reactions in these patients, and they have been able to tolerate the course of treatment much more satisfactorily. No ill effects from the use of hydrocortisone have so far been noted. A few of our patients have also received streptomycin as additional cover against possible tuberculosis or for a coexisting tuberculous infection, and there is no evidence among these patients that the use of streptomycin has been responsible for any deterioration. There is often increase in weight and decrease in sputum and cough in treated patients. It may be suggested that all cases in which a diagnosis of histoplasmosis has been reached by either a positive sputum culture or the isolation of the organism from surgical specimens should perhaps be given a course of amphotericin B. Those with a high complement-fixation reaction only may also benefit from therapy. We have had a few patients who were operated on without amphotericin B cover, and, as has been pointed out, a post-operative air space $\overrightarrow{\vec{S}}$ has developed which responded satisfactorily to $\frac{?}{0}$ amphotericin B, so that cover for operation on $\frac{\mathrm{C}}{\mathrm{O}}$ these cases is an additional advantage.

Tolerance to intravenous amphotericin $B$ is $\vec{\nabla}$ variable and side-effects often noticeable. Many of

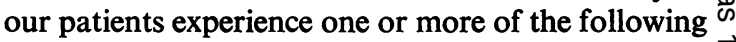
effects during some stage of the treatment: nausea, $\vec{O}$ dizziness, headache, chills, fever, thrombophlebitis. $\overrightarrow{\vec{H}}$ We have used acetylsalicylic acid, thorazine, and $\vec{\sigma}$ intravenous hydrocortisone to counteract these side- $\stackrel{P}{\vec{P}}$ effects, and have found the latter particularly useful. $\vec{x}$ It has also been noted that, if therapy is temporarily $\vec{v}$ discontinued on a particular patient, side-effects are $\dot{\omega}$ more noticeable on resumption of therapy. $\mathcal{O}$ Abnormal results of non-protein nitrogen, blood urea nitrogen and sulphobromophthalein examina- $\stackrel{\supset}{-}$ tions are often seen, though these return to normal $\vec{\omega}$ after cessation of treatment.

There is apparently room for progress in labora- $\frac{\mathbb{D}}{\vec{D}}$ tory diagnostic methods as some centres can more $\frac{3}{0}$ readily recognize the organisms than others. Sweany, Gorelick, Coller, and Jones (1958) report $\vec{\varphi}$ that the organisms are more likely to be found in adjacent caseous nodes than in the actual cavity wall. Thus it becomes important what section one submits for these special examinations. The favour the Gomori methenamine silver stainims technique, and they use polarized light as a counter $\frac{\square}{\varnothing}$ check to eliminate artefacts. Saliba and Beatty $\cong$ (1960) have shown that a histological diagnosis can $\overrightarrow{0}$ often be misleading in surgical cases. Tissue has 3 been reported as typical of "tuberculosis" when pre-operatively positive cultures for Histoplasma capsulatum were available. However, as a control, sections from these specimens were also submitted to a particular centre where the presence of Histoplasma capsulatum was readily established. Something to this effect has been noted by Segal, Starr, and Weed (1959), who identified structures resembling histoplasma or coccidioides in $55.9 \%$ of a group of surgically excised "pulmonary granulomas." "

Judging by the source of histoplasmosis infection, it is possible that with greater scrutiny of bacterio- $N$ logically unproved "tuberculous" patients more and more cases may be recognized in many other countries where sporadic cases have been reported. $\omega$ More knowledge is being accumulated about this disease and its management. Treatment of the chronic form with amphotericin is helpful but $\Phi_{\infty}^{\Phi}$ certainly not very dramatic in results. It is likely ${ }^{+}$ that there will be more effective therapy in the future. 


\section{SUMMARY}

A selection of 12 cases of pulmonary histoplasmosis is presented. In 11 cases $H$. capsulatum was isolated from sputum or tissue sections. The remaining case had a highly positive complementfixation, but negative sputum cultures. Radiologically these cases presented cavitation, destroyed lung, nodular or miliary disease, enlarged hilar glands, or infiltration.

The prevalence of the condition is briefly discussed. Treatment with amphotericin B has been found helpful in these cases, though no dramatic radiographic improvement was noted in this chronic type of the disease. It is suggested that awareness of the prevalence of the condition and a closer scrutiny of bacteriologically negative " tuberculous" cases may bring more cases to light.

\section{BIBLIOGRAPHY}

Bunnell, I. L., and Furcolow, M. L. (1948). Publ. Hlth Rep. (Wash.),

Conrad, F. G., Saslaw, S., and Atwell, R. J. (1959). A.M.A. Arch. intern. Med. 104, 692 .

Darling, S. T. (1906). J. Amer. med. Ass., 46, 1283.

Furcolow, M. L. (1958). G.P. (Kansas), 18, Oct. p. 117.

Johnson, H. E., and Batson, R. (1948). Dis. Chest, 14, 517.

Rubin, H., Furcolow, M. L., Yates, J. L., and Brasher, C. A. (1959). Amer. J. Med., 27, 278.

Saliba, A., Beatty, O. A., and Pakalns, A. (1960). Amer. Rev. Tuberc. 81,709

Tch (1960). J. Amer. med. Ass., 173, 902.

.

Segal, E. L., Starr, G. F., and Weed, L. A. (1959). J. Amer. med. Ass., $170,515$.

Sweany, H. C., Gorelick, D., Coller, F. C., and Jones, J. L. (1958), Dis. Chest, 34, 119 and 257.

Yates, J. L., Atay, M. N., Langeluttig, H. V., Brasher, C. A., and Furcolow, M. L. (1960). Ibid., 37, 144 